\title{
Controllable Frequency Entanglement via Auto-Phase-Matched Spontaneous Parametric Down-Conversion
}

\author{
Zachary D. Walton $*$ Mark C. Booth, Alexander V. Sergienko, Bahaa E. A. Saleh, and Malvin C. Teich \\ Quantum Imaging Laboratory, Department of Electrical \&s Computer Engineering, \\ Boston University, 8 Saint Mary's Street, Boston, Massachusetts 02215-2421
}

\begin{abstract}
A new method for generating entangled photons with controllable frequency correlation via spontaneous parametric down-conversion (SPDC) is presented. The method entails initiating counterpropagating SPDC in a single-mode nonlinear waveguide by pumping with a pulsed beam perpendicular to the waveguide. The method offers several advantages over other schemes, including the ability to generate frequency-correlated photon pairs regardless of the dispersion characteristics of the system.
\end{abstract}

PACS numbers: 42.65.-k, 42.50.Dv, 03.67.-a, 03.65.Ud

Spontaneous parametric down-conversion (SPDC) is a convenient process for generating pairs of photons that are entangled in one or more of their respective degrees of freedom (direction, frequency, polarization). This entanglement can be used to demonstrate counter-intuitive features of quantum mechanics and to implement the growing suite of quantum information technologies [1]. In a typical down-conversion experiment, a photon from a monochromatic pump beam decays into two photons (often referred to as signal and idler) via interaction with a nonlinear optical crystal. While the signal and idler may be broadband individually, conservation of energy requires that the sum of their respective frequencies equals the single frequency of the monochromatic pump. This engenders frequency anti-correlation in the down-converted beams. Aside from the frequency-anticorrelated case, the frequency-correlated and frequencyuncorrelated cases were also investigated theoretically by Campos et al. in 1990 [2]. At that time, neither a method of creating these novel states nor a practical application of the states was known.

Two developments in quantum information theory have renewed interest in these generalized states of frequency correlation. First, quantum information processes requiring the synchronized creation of multiple photon pairs have been devised, such as quantum teleportation [3] and entanglement swapping [4]. The requisite temporal control can be achieved by pumping the crystal with a brief pulse. The availability of pump photons of differing frequencies relaxes the strict frequency anticorrelation in the down-converted beams [5]. Second, applications such as entanglement-enhanced clock synchronization [6] and one-way auto-compensating quantum cryptography [7] have been introduced that specifically require frequency correlation, as opposed to the usual frequency anti-correlation.

Methods for preparing these novel states of frequency

\footnotetext{
*Electronic address: walton@bu.edu Quantum Imaging Laboratory homepage: http://www.bu.edu/qil
}

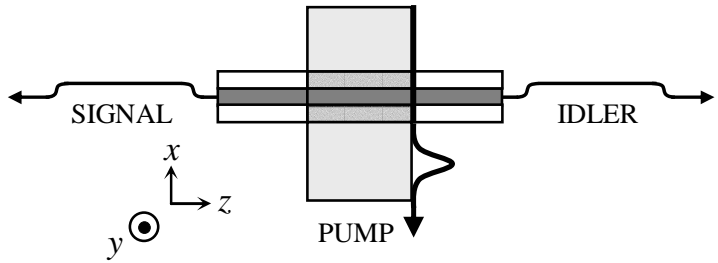

FIG. 1: A schematic of auto-phase-matched SPDC, a new method for generating entangled-photon pairs with controllable frequency correlation. The z-polarized pulsed pump beam initiates counter-propagating y-polarized SPDC in the single-mode nonlinear waveguide. The joint spectrum of the down-converted beams is controlled by the spatial and temporal characteristics of the pump beam, as described in the text.

correlation have emerged as well. Keller and Rubin were first to observe that when a specific relationship between the group velocities of the pump and downconverted beams holds, the down-converted photons are anti-correlated in time [5]. Using a first-order Taylor approximation of the relevant dispersion curves, they provided two examples of bulk nonlinear crystals that satisfied this relationship when used in a collinear type-II configuration (signal and idler orthogonally polarized). Erdmann et al. pointed out that the time-anti-correlated state described by Keller and Rubin entails frequency correlation (which can be seen immediately by Fourier duality), and further emphasized that perfect frequency correlation requires an infinite crystal, just as perfect frequency anti-correlation requires a pump with an infinite coherence length 8]. More recently, Giovannetti et al. demonstrated the feasibility of frequency-correlated down-conversion in a periodically-poled nonlinear crystal [9] and presented a formalism for parameterizing the space of states between the cases of perfect frequency correlation and perfect frequency anti-correlation [10].

In this article, we present a new method for obtaining controllable frequency entanglement that has distinct advantages over the previously proposed methods. Our method entails initiating type-I SPDC (signal and idler 
identically polarized) in a single-mode nonlinear waveguide by pumping with a pulsed beam perpendicular to the waveguide (see Fig. 11). The down-converted photons emerge from opposite ends of the waveguide with a joint spectrum that can be varied from frequency anticorrelated to frequency correlated by adjusting the temporal and spatial characteristics of the pump beam. The primary advantage of this method is that the limiting cases of perfect frequency correlation and perfect frequency anti-correlation can be obtained regardless of the dispersion relation of the waveguide. Thus, we refer to the method as auto-phase-matched. It is well known that the frequency-anti-correlated case is achievable regardless of the dispersion relations in a collinear configuration with a monochromatic pump and a thin bulk crystal; however, the frequency-correlated case has hitherto been associated with a constraint on the dispersion relations (cf. the "group velocity matching" condition introduced in Ref. 5]). The geometry we propose restores the symmetry between the two cases by ensuring the appropriate phase-matching regardless of the dispersion relation of the waveguide.

This article is organized as follows. First, we write the output state of the SPDC produced in our new configuration and provide an estimate of the conversion efficiency. Second, we analyze the state using a Franson interferometer [11], which illustrates the duality between frequency correlation and anti-correlation. Third, we quantify our method's advantage by comparing the visibility achieved in a Franson interferometer by the frequency-correlated, collinear configuration described in Ref. 9] with the visibility achieved in our counter-propagating configuration.

The transverse-pump, counter-propagating geometry depicted in Fig. 1 has been noted as a promising source of entangled-photon pairs for both type-I [12] and typeII 13. SPDC. The most obvious advantages of this geometry over a collinear geometry pertain to the separation of the three interacting beams. In a transverse-pump, counter-propagating geometry, all three beams are traveling in different directions. Thus, the usual techniques for filtering the pump beam from the down-conversion and separating the down-converted beams at a beamsplitter are unnecessary.

The investigations in Refs. 12, 13] were limited to the case of a monochromatic pump beam. There are two primary advantages of pumping with a broadband beam perpendicular to the waveguide and arranging for type-I down-conversion. First, the dispersion relation for the pump beam plays no role in the phase-matching analysis, since the waveguide ensures phase-matching in the transverse direction. Second, the counter-propagating, identically-polarized signal and idler fields will be phasematched in the long-crystal limit only if they have equal and opposite propagation vectors, a condition which entails equal frequency. Thus, the bandwidth of the pump determines the allowable range of the sum frequency of the signal and idler, and the longitudinal length of the illuminated portion of the crystal determines the allowable range of the difference frequency.

We assume that the nonlinear coefficient and the propagation constants vary sufficiently slowly with frequency that they may be taken outside any frequency integrals in which they appear as integrand prefactors. Furthermore, we assume that the waveguide is long compared to the width of the pump beam such that the interaction length is controlled by the pump beam profile along the z-axis (see Fig. 1). Following the derivation in Ref. 12] of the quantum state of a counter-propagating photon pair, we have

$$
|\Psi\rangle \propto \iint d \omega_{l} d \omega_{r} \tilde{E}_{t}\left(\omega_{l}+\omega_{r}\right) \tilde{f}_{z}\left[\Delta \beta\left(\omega_{l}, \omega_{r}\right)\right]\left|\omega_{l}\right\rangle_{l}\left|\omega_{r}\right\rangle_{r}
$$

where $\tilde{E}_{t}(\omega)$ and $\tilde{f}_{z}(\Delta \beta)$ are the respective Fourier transforms of the temporal and spatial functions describing the pump beam $E_{p}(t, z)=E_{t}(t) f_{z}(z), \Delta \beta\left(\omega_{l}, \omega_{r}\right)=$ $\beta\left(\omega_{l}\right)-\beta\left(\omega_{r}\right)$ is the difference in the waveguide propagation constant evaluated at $\omega_{l}$ and $\omega_{r}$, and $|\omega\rangle_{l(r)}$ denotes a single photon at frequency $\omega$ moving to the left(right).

To investigate the dependence of $|\Psi\rangle$ on the characteristics of the pump, we choose Gaussian profiles in space and time for the pump pulse, such that $\tilde{E}_{t}(\omega) \propto e^{-\frac{1}{2}(\omega \tau)^{2}}$ and $\tilde{f}_{z}(\Delta \beta) \propto e^{-\frac{1}{2}(\Delta \beta W)^{2}}$, where $\tau$ and $W$ are the duration and width (along the $z$-axis in Fig. 1) of the pump pulse, respectively. In the limit of a monochromatic pump $(\tau \rightarrow \infty)$ with finite spatial extent, $\tilde{E}_{t}\left(\omega_{l}+\omega_{r}\right)$ is sharply peaked around the pump center frequency. Thus, the sum frequency of the signal and idler is fixed. This is the familiar frequency-anti-correlated case that is readily achievable in thin bulk crystals. In the limit of a finiteduration pump pulse of infinite spatial extent $(W \rightarrow \infty)$, $\tilde{f}_{z}\left[\Delta \beta\left(\omega_{l}, \omega_{r}\right)\right]$ is sharply peaked around $\Delta \beta=0$. Thus, photon pairs for which $\omega_{l} \approx \omega_{r}$ predominate. This is the frequency-correlated case that has hitherto only been achieved by imposing a group velocity matching condition.

The efficiency of this geometry in an GaAs-based waveguide of length $1 \mathrm{~mm}$ and transverse dimension 3 $\mu \mathrm{m}$ is calculated in Ref. [13] to range between $10^{-9}$ and $10^{-11}$ depending on the transverse profile of the waveguide. These figures compare favorably with the SPDC efficiencies achieved in more conventional bulk-crystal configurations (e.g., $10^{-13}$ in Ref. [14]), though they are still several orders of magnitude less than that achieved in periodically poled lithium niobate waveguides (e.g., $10^{-6}$ in Ref. [15]).

The Franson interferometer [1] is a natural tool for distinguishing frequency correlation and frequency anticorrelation. When the two delays $\left(\tau_{1}\right.$ and $\left.\tau_{2}\right)$ are equal to within the reciprocal bandwidth of down-conversion, coincidence detections can be associated with indistinguishable pair-creation events (see Fig. 2A). If the downconverted photons are correlated in time (anti-correlated in frequency), the short-short two-photon amplitude interferes with the long-long amplitude (see Fig. 23). If the down-converted photons are anti-correlated in time 


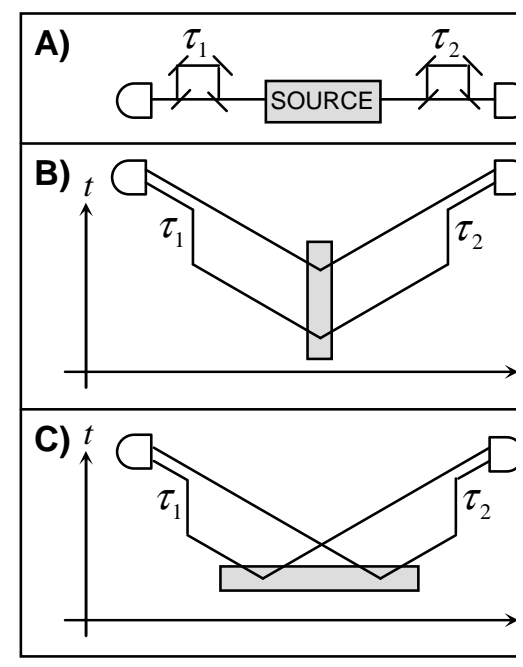

FIG. 2: The Franson interferometer (A) and of indistinguishability it can bring about ( $\mathrm{B}$ a picts the indistinguishability in time of creation pair, and C) depicts indistinguishability in $\mathrm{p}$ ation of the photon pair. These two cases are text to correspond to frequency-anti-correlatec and frequency-correlated photon pairs, respect

(correlated in frequency), the short-long am feres with the long-short amplitude (see F duality between these two cases can be $\mathrm{s}$ paring the loci of indistinguishable pair-cr in the spacetime diagrams of Fig. $2 \mathrm{~B}$ and frequency-anti-correlated case depicted in I from the coherent superposition of pair-cr at a fixed position over a range of time frequency-correlated case depicted in Fig. 2 the coherent superposition of pair-creatior fixed time over a range of positions. Note $t$ interference visibility decreases in both cas approaches the reciprocal bandwidth of dow the relative phase between the interfering as pends on $\tau_{1}+\tau_{2}$ in the frequency-anti-cos and on $\tau_{1}-\tau_{2}$ in the frequency-correlated ,

In Fig. [3] we plot the probability of coinc Franson interferometer for the aforementis cases of the two-photon source: perfect fre correlation $(\tau \rightarrow \infty, W \rightarrow$ finite $)$, and perfe anti-correlation $(\tau \rightarrow$ finite, $W \rightarrow \infty)$. The of $\tau$ and $W$ are chosen such that the bandw. conversion is $\omega_{p} / 10 \mathrm{in}$ each case. The fourth in the $\tau_{1} \approx \tau_{2} \gg 10 / \omega_{p}$ region show that interferometer clearly distinguishes the tw modulation is in the $\Delta \tau_{1}=\Delta \tau_{2}$ direction $\mathrm{t}$. anti-correlated case and in the $\Delta \tau_{1}=-\Delta \tau$ the frequency-correlated case.

By establishing the signature of the perfe correlated state (the fourth-order fringes in are able to compare the performance of methods designed to produce this state. Sp
A) FREQUENCY ANTI- CORRELATED

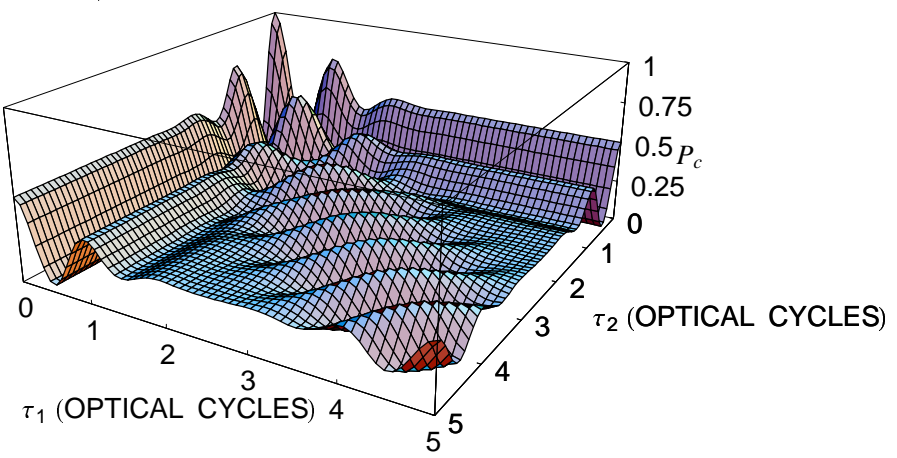

B) FREQUENCY CORRELATED

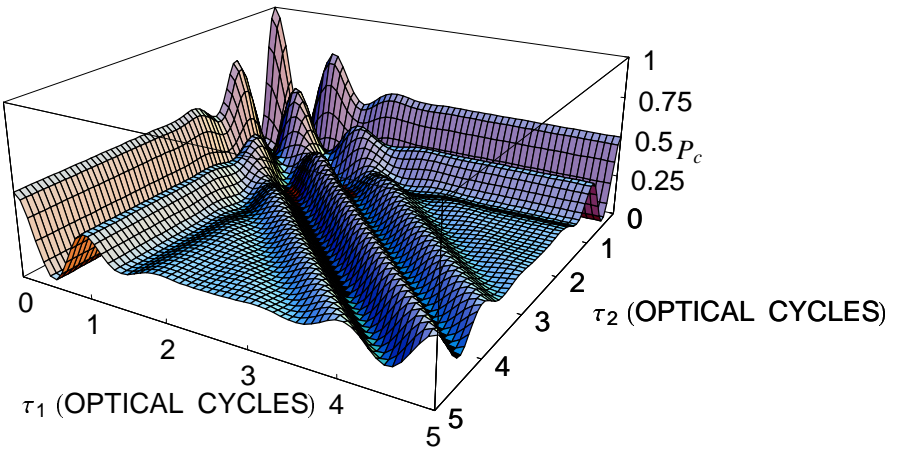

FIG. 3: The probability of coincidence when frequency-anticorrelated (A) and frequency-correlated (B) states are analyzed with a Franson interferometer (see Fig. 2). The downconverted beams have center frequency $\omega_{p} / 2$ and bandwidth $\omega_{p} / 10 . \tau_{1}$ and $\tau_{2}$ are in units of optical cycles at the center frequency of downconversion.

visibility of the fringes in Fig. $3 \mathrm{~B}$ provides a measure of the quality of the frequency-correlated state. In Fig. 目 we plot a numerical calculation of the visibility achieved by the source described in Ref. [9] (thin line) and that achieved by our auto-phase-matched method (thick line) in a GaAs-based waveguide, for a range of interaction lengths. In the method of Ref. [9] the interaction length is the thickness of the crystal, while in our method the interaction length is the width of the pump beam in along the z-axis (see Fig. 1). In order to minimize the complicating effect of second-order interference, the visibility is calculated at the delay offset $\left(\tau_{1}, \tau_{2}\right)=(4 / \sigma, 4 / \sigma)$ where $\sigma$ is the bandwidth of down-conversion.

While the fourth-order visibility in Fig. $3 \mathrm{~B}$ is 0.5 , we have scaled the visibilities to range between 0 and 1 in Fig. 4 since methods exist for restoring maximal visibility in the Franson interferometer. The first method put forward involves time-gating the detectors [17]. For that method to work with the source described here, the waveguide would have to be much longer $(>5 \mathrm{~cm})$ than those in current experimental designs $(\sim 3 \mathrm{~mm}$ in Ref. [18]). A more promising approach for restoring visibility exploits polarization entanglement, as demon- 


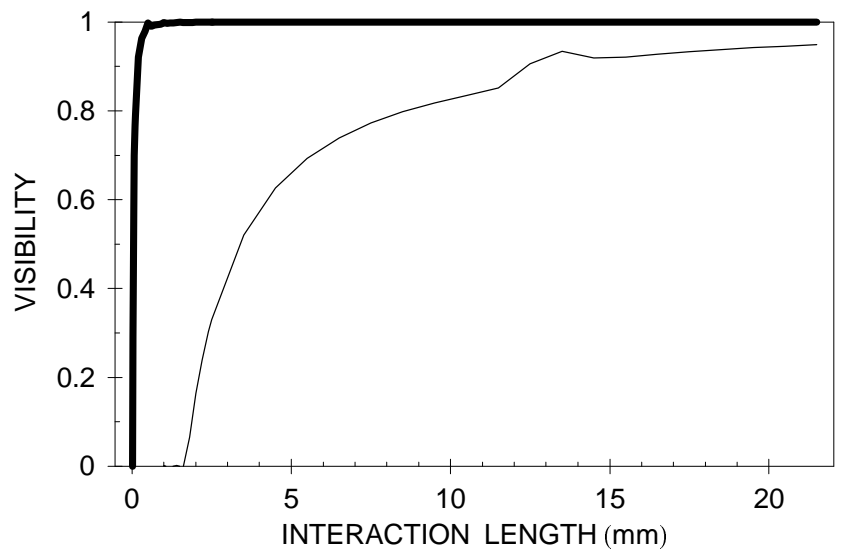

FIG. 4: Numerical calculation of the fourth-order fringe visibility seen in a Franson interferometer when the perfect source of frequency-correlated photon pairs is approximated by the method described in Ref. [9] (thin line) and by the auto-phasematched method described in the text (bold line). The plot depicts the effect of changing the interaction length of the nonlinear process while holding the bandwidth of the pump fixed.

strated in Ref. [19]. At the end of this paper we discuss the natural way in which the source described here enables concurrent polarization and frequency entanglement.

The parameters of the source described in Ref. [9] are as follows: periodically-poled potassuium titanyl phosphate (PPKTP) with a poling period of $47.7 \mu \mathrm{m}$, pump wavelength of $790 \mathrm{~nm}$, pump bandwidth of $3 \mathrm{THz}$, and collinear propagation along the crystal's $x$ axis with the signal $z$-polarized and the pump and idler $y$-polarized. For our new geometry, we consider a GaAs slab waveg- uide which is $3 \mu \mathrm{m}$ in the transverse dimension and configured as depicted in Fig. 1 with the pump $z$-polarized and the signal and idler polarized in the $x-y$ plane. Since both schemes rely on a long-crystal phase-matching condition, it is not surprising that the visibility of each increases with increasing interaction length; however, while the deleterious influence of higher-order terms in the crystal dispersion relations is exacerbated as the crystal length is increased in the method of Ref. [9], our autophase-matched approach provides an increasingly close approximation to the desired state, regardless of the dispersion relations.

Finally, it is worth noting that our source may be used to create photon pairs that are entangled in polarization. In Fig. 11 we were only concerned with downconverted photons polarized along the y-axis. If the pump beam is polarized along the z-axis and we use a material with the symmetry properties of GaAs which has $\chi_{z x y}^{(2)}=\chi_{z y x}^{(2)}$ and $\chi_{z x x}^{(2)}=\chi_{z y y}^{(2)}=0$ [16], we will get a counter-propagating polarization-entangled state $(|H V\rangle+|V H\rangle$ directly from the crystal [20]. Furthermore, we can obtain this polarization-entanglement while independently controlling the frequency entanglement by manipulating the pump beam, as previously described.

\section{Acknowledgments}

This work was supported by the National Science Foundation; the Center for Subsurface Sensing and Imaging Systems (CenSSIS), an NSF Engineering Research Center; the Defense Advanced Research Projects Agency (DARPA); and the David and Lucile Packard Foundation.
[1] M. A. Nielsen and I. L. Chuang, Quantum Computing and Quantum Information (Cambridge, New York, 2001).

[2] R. A. Campos, B. E. A. Saleh, and M. C. Teich, Phys. Rev. A 42, 4127 (1990).

[3] C. H. Bennett, G. Brassard, C. Crépeau, R. Jozsa, A. Peres, and W. K. Wootters, Phys. Rev. Lett. 70, 1895 (1993).

[4] J. W. Pan, D. Bouwmeester, H. Weinfurter, and A. Zeilinger, Phys. Rev. Lett. 80, 3891 (1998).

[5] T. E. Keller and M. H. Rubin, Phys. Rev. A 56, 1534 (1997).

[6] V. Giovannetti, S. Lloyd, and L. Maccone, Nature 412, 417 (2002).

[7] Z. Walton, A. F. Abouraddy, A. V. Sergienko, B. E. A. Saleh, and M. C. Teich, quant-ph/0207167 (2002).

[8] R. Erdmann, D. Branning, W. Grice, and I. A. Walmsley, Phys. Rev. A 62, 53810 (2000).

[9] V. Giovannetti, L. Maccone, J. H. Shapiro, and F. N. C. Wong, Phys. Rev. Lett. 88, 183602 (2002).

[10] V. Giovannetti, L. Maccone, J. H. Shapiro, and F. N. C.
Wong, Phys. Rev. A 66, 43813 (2002).

[11] J. D. Franson, Phys. Rev. Lett. 62, 2205 (1989).

[12] M. C. Booth, M. Atatüre, G. Di Giuseppe, B. E. A. Saleh, A. Sergienko, and M. C. Teich, Phys. Rev. A 66, 23815 (2002).

[13] A. De Rossi and V. Berger, Phys. Rev. Lett. 88, 043901 (2002).

[14] T. Jennewein, C. Simon, G. Weihs, H. Weinfurter, and A. Zeilinger, Phys. Rev. Lett. 84, 4729 (2000).

[15] S. Tanzilli, H. D. Riedmatten, W. Tittel, H. Zbinden, P. Baldi, M. D. Micheli, D. B. Ostrowsky, and N. Gisin, Electron. Lett. 37, 26 (2001).

[16] A. Yariv, Quantum Electronics (John Wiley \& Sons, New York, 1988), 3rd ed.

[17] J. Brendel, E. Mohler, and W. Martienssen, Phys. Rev. Lett. 66, 1142 (1991).

[18] A. De Rossi, V. Berger, M. Calligaro, G. Leo, V. Ortiz, and X. Marcadet, Appl. Phys. Lett. 79, 3758 (2001).

[19] D. V. Strekalov, T. B. Pittman, A. V. Sergienko, Y. H. Shih, and P. G. Kwiat, Phys. Rev. A 54, R1 (1996).

[20] The relative phase between the two terms $(|H V\rangle$ and 
$|V H\rangle)$ in the superposition is unity since GaAs is not birefringent. 